\title{
1 Generating heterokaryotic cells via bacterial cell-cell fusion
}

3 Shraddha Shitut ${ }^{1,2,3}$, Meng-Jie Shen ${ }^{2}$, Bart Claushuis ${ }^{3}$, Rico J. E. Derks ${ }^{4}$, Martin

4 Giera $^{4}$, Daniel Rozen ${ }^{3}$, Dennis Claessen ${ }^{3}$, Alexander Kros $^{2}$

$6{ }^{1}$ Origins Centre, Groningen, the Netherlands

72 Dept. Supramolecular \& Biomaterials chemistry, Leiden Institute of Chemistry, Leiden

8 University, the Netherlands

$9{ }^{3}$ Institute of Biology, Leiden University, the Netherlands

$10 \quad{ }^{4}$ Center for Proteomics and Metabolomics, Leiden University Medical Center, the

11 Netherlands

13 Correspondence to: s.s.shitut@lic.leidenuniv.nl (SS),

14 d.claessen@biology.leidenuniv.nl (DC), a.kros@chem.leidenuniv.nl (AK)

\section{Abstract}

17 Cell-cell fusion is fundamentally important for tissue repair, virus transmission, and genetic recombination, among other functions. Fusion has been mainly studied in eukaryotic cells and lipid vesicles, while cell-cell fusion in bacteria is less well characterized, due to the cell wall acting as a fusion-limiting barrier. Here we use cell wall-deficient bacteria to investigate the dynamics of cell fusion in bacteria that replicate without their cell wall. Stable, replicating cells containing differently labeled chromosomes were successfully obtained from fusion. We find that the rate of cell-cell fusion depends on the fluidity of cell membranes. Furthermore, we show that not only the efficiency but also the specificity of cell-cell fusion can be controlled via a pair of synthetic membrane-associated lipopeptides. Our results provide a molecular handle to understand and control cell-cell fusion to generate heterokaryotic cells, which was an important step in the evolution of protocells and of increasing importance for the design of synthetic cells. 


\section{Introduction}

The structural and functional complexity of modern bacterial cells evolved gradually over hundreds of millions of years from much simpler enclosed protocells (Szostak, Bartel, and Luisi 2001). These early cells are thought to have resembled selforganizing lipid spheres containing stable catalytic actitivity or primitive metabolism (Monnard and Deamer 2002), but lacking a rigid cell wall. Lipid vesicles are widely used to study the behavior of protocells because they are capable of compartmentalization as well as growth and proliferation (Szostak, Bartel, and Luisi 2001; Adamala and Luisi 2011). Proliferation of such vesicles involves dramatic shape perturbations, such as fission, tubulation, and vesiclulation, which likely preceded the coordinated cell division of modern walled bacteria (Svetina 2009; Hanczyc and Szostak 2004). However, because lipid vesicles are inherently limited in terms of their internal cytoplasmic complexity, consisting of only minimal catalytic components, new models are needed that more closely resemble protocells to effectively study their early evolution (Briers et al. 2012; Errington et al. 2016). This is particularly needed to examine mechanisms and genetic consequences of cell fusion, an early mechanism of microbial horizontal gene transfer (Kotnik 2013; Soucy, Huang, and Gogarten 2015; Naor and Gophna 2013).

Cell fusion has been studied in many different eukaryotic cell types (Chen et al. 2007) and is crucial for tissue repair and regeneration, phenotypic diversity, viral transmission and recombination (Ogle, Cascalho, and Platt 2005). The process of fusion proceeds via several steps: cell adhesion, recognition of cell surface components, membrane remodelling and in some cases nuclear fusion (Zito et al. 2016). These processes are highly influenced by lipid-lipid interactions (Chernomordik, Kozlov, and Zimmerberg 1995) which have been studied using coarse grained lipid models and lipid vesicles (Smeijers et al. 2006; Marrink and Mark 2003). Fusion in eukaryotic cells is induced via SNARE proteins that form complexes to bridge together membranes by pulling cells close to each other (Hanson, Heuser, and Jahn 1997). The potential for SNARE proteins, or related tools that bridge membranes, to facilitate bacterial fusion have not yet been explored. Studying cell/membrane fusion in eukaryotes and lipid vesicles have unravelled details of the molecular mechanism of membrane fusion; however these systems are highly divergent in terms of cellular and molecular complexity and are not representative of bacterial fusion, which may be common in species lacking a cell wall. 
Many bacterial species can transiently shed their cell wall when exposed to environmental stressors like cell wall targeting antibiotics and osmotic stress (Claessen and Errington 2019). When these stressors are removed, wall-deficient cells can rebuild their cell wall and revert to their walled state. Alternatively, prolonged exposure to these stressors can lead to the formation of so-called L-forms, which can efficiently propagate without their wall (Mercier, Kawai, and Errington 2014; Innes and Allan 2001; Glover, Yang, and Zhang 2009; Studer et al. 2016). Much like lipid vesicles, L-form growth and division is regulated by physicochemical forces that deform the cell membrane, leading to an irregular assortment of progeny cells. However, L-forms contain the sophisticated machinery of modern cells which is lacking in protocell models based on giant lipid vesicles (Briers et al. 2012). This makes them suitable to understanding the dynamics and consequences of cellular fusion, as well as to identify factors that affect this process.

In this study we show that fusion between L-form cells is a dynamic process whose frequency is dependent on the age of the bacterial culture; this, in turn, is determined by the fluidity of the cell membrane, which we confirm by chemically manipulating membrane fluidity. In addition, we demonstrate for the first time that complementary lipidated coiled coil lipopeptides (structurally similar to SNARE proteins) increase the efficiency and specificity of cell-cell fusion. Importantly, fusants resulting from this process are viable and express markers from both parental chromosomes. This opens up avenues to design complex heterokaryotic/hybrid cells that have potential not only to answer questions on evolution of complexity but also enable novel applications in biotechnology.

\section{Results}

\section{A dual marker system for identifying cell-cell fusion} In order to study cell-cell fusion, we created two fluorescent strains by integrating plasmids $\mathrm{pGreen}$ or $\mathrm{pRed} 2$ into the attB site in the genome of an L-form derivative of the actinobacterium K. viridifaciens (Fig. 1A). The strain carrying pGreen constitutively expresses EGFP and is apramycin resistant, while the strain carrying pRed2 constitutively expresses mCherry and is hygromycin resistant (Fig. 1A). We first confirmed resistance to these antibiotics by determining the susceptibility of each strain to both antibiotics (Fig. 1B, supplementary fig. 1A). The strain expressing resistance to apramycin (referred to as AG [for Apramycin-Green]) was able to grow 

[for Hygromycin-Red]) could grow at $100 \mu \mathrm{g} \mathrm{mL}-1$ hygromycin. Resistance to one antibiotic did not provide cross-resistance to the other. Confirmation of the fluorescence reporters was obtained via microscopy with cytoplasmic eGFP detected in the AG strain and mCherry detected in the HR strain (Fig. 1C).

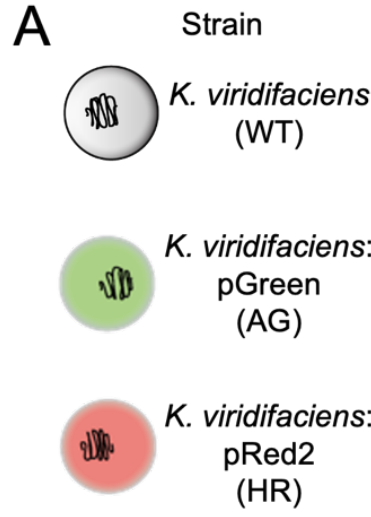

109

110

111

112

113

114

115

116

117

118

119

120

121

122

123

124

125

126

127

128

130

131

132
B
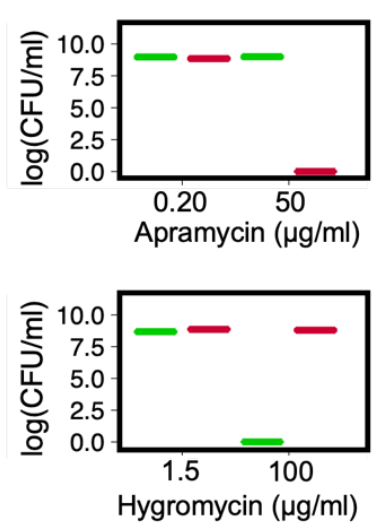
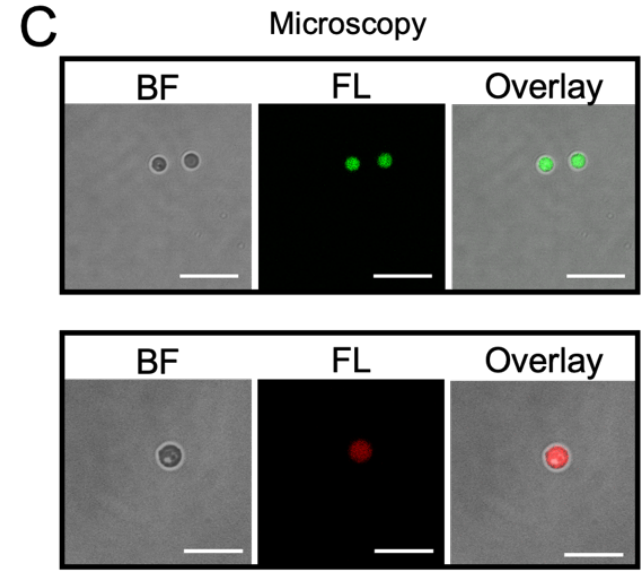

Figure 1. L-forms used in the study. (A) The wildtype Kitasatospora viridifaciens delta L-form strain was genetically modified to either express apramycin resistance and green fluorescence (AG) or hygromycin resistance and red fluorescence (HR). Each reporter pair (antibiotic resistance+ fluorescence gene) was introduced via a plasmid using the $\phi C 31$ integration system. (B) Antibiotic susceptibility testing showed growth of the desired strain at $50 \mu \mathrm{g} / \mathrm{ml}$ apramycin for $A G$ and $100 \mu \mathrm{g} / \mathrm{ml}$ hygromycin for HR. (C) Visual confirmation of fluorescence reporters using microscopy indicated a positive signal in the green channel for AG and in the red channel for HR. Scale bar represents $10 \mu \mathrm{m}$.

\section{Fusion of L-form using centrifugation and PEG}

L-forms show structural resemblance to protoplasts that are often used for genome reshuffling in plants and bacteria via the process of cell-cell fusion. After fusion these protoplasts can revert back to their walled state. To analyse the ability of L-forms to fuse, we tested some commonly used methods for protoplast fusion (Kieser et al. 2000; Baltz and Matsushima 1981; Gokhale, Puntambekar, and Deobagkar 1993) namely, mechanical force induced fusion via centrifugation and PEG-mediated fusion (Fig. 2). Non-specific fusion between $A G$ and HR strains via centrifugation or PEG could result in three different genotypes: $A G / H R, A G / A G$ and HR/HR. However, genetically identical fusants (AG/AG and HR/HR) would not grow on selection plates containing both antibiotics (supplementary fig. 1B). Fusion frequencies determined by growth on both antibiotics are therefore an underestimate of true fusion rates. Centrifuging mixtures of $A G$ and $H R$ at $500 \mathrm{xg}$ resulted in the highest fusion efficiency (1.5 in $10^{5}$ cells); however, the pellet formed in this case was difficult to handle. Increasing centrifugation to $1000 \mathrm{xg}$ reduced the fusion efficiency to less than 1 fused 
cell per $10^{5}$ cells, and no fusion was observed at speeds above $6000 \mathrm{xg}$ due to cell

134 Iysis (Fig. 3A). The fusion efficiency in the presence of PEG was highest at $10 \mathrm{w} \%$

135 PEG with 1 fused cell per $10^{5}$ cells (Fig. 3B). Higher PEG concentrations, such as 50

$136 \mathrm{w} \%$ that is commonly used for protoplast fusion, caused dramatic cell lysis, suggesting

137 that the membrane composition of L-forms is different from protoplasts.

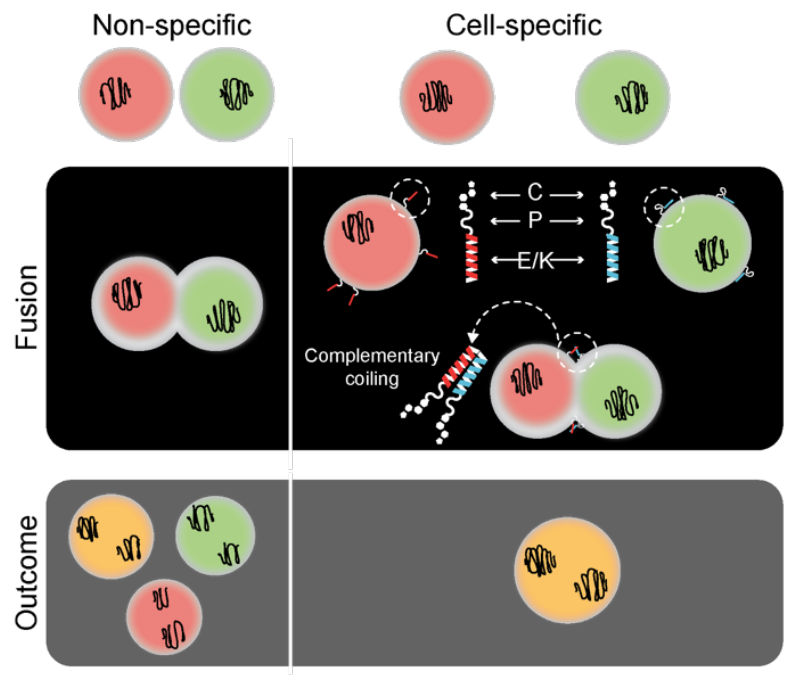

Figure 2. Schematic of L-form fusion. Fusion was obtained by two types of methods: non-specific (centrifugation, poly(ethyleneglycol)-PEG) and cell-specific (coiled coil lipopeptides). The process of fusion (black box) and the outcome (grey box) differs in both cases. For non-specific fusion the membranes come together by dehydration induced by PEG or physical centrifugal force. In the case of coiled coil lipopeptides (CPE and $\mathrm{CPK}$ ), they dock in the membrane using the cholesterol anchor and pull together opposing membranes upon complementary coiling. This complementarity results in fusion of only oppositely labelled cells unlike that in the non-specific methods.

To verify that the cells growing on plates with both antibiotics (supplementary fig. 1B) were true fusants, we used microscopy. A small patch of biomass growing on media with both antibiotics was imaged using fluorescence microscopy (Fig. 3C). The percent of pixels that were double labelled (i.e. containing both green and red emission) was higher for cells that had undergone fusion via PEG (21.52\%) compared to centrifugal force $(11.92 \%)$. These patches of double labelled cells indicate the presence and subsequent expression of both sets of marker ( $A G$ and HR). The presence of green and red patches in the colonies can be attributed to the fact that the polyploid L-forms may consist of an unequal ratio of the two chromosome types. An unequal ratio and expression of markers can lead to a predominantly green (more AG than HR) or red (more HR than AG) colony appearance. Taken together these results show that cell-cell fusion of L-forms is possible and that the resulting colonies contain both chromosomes. 

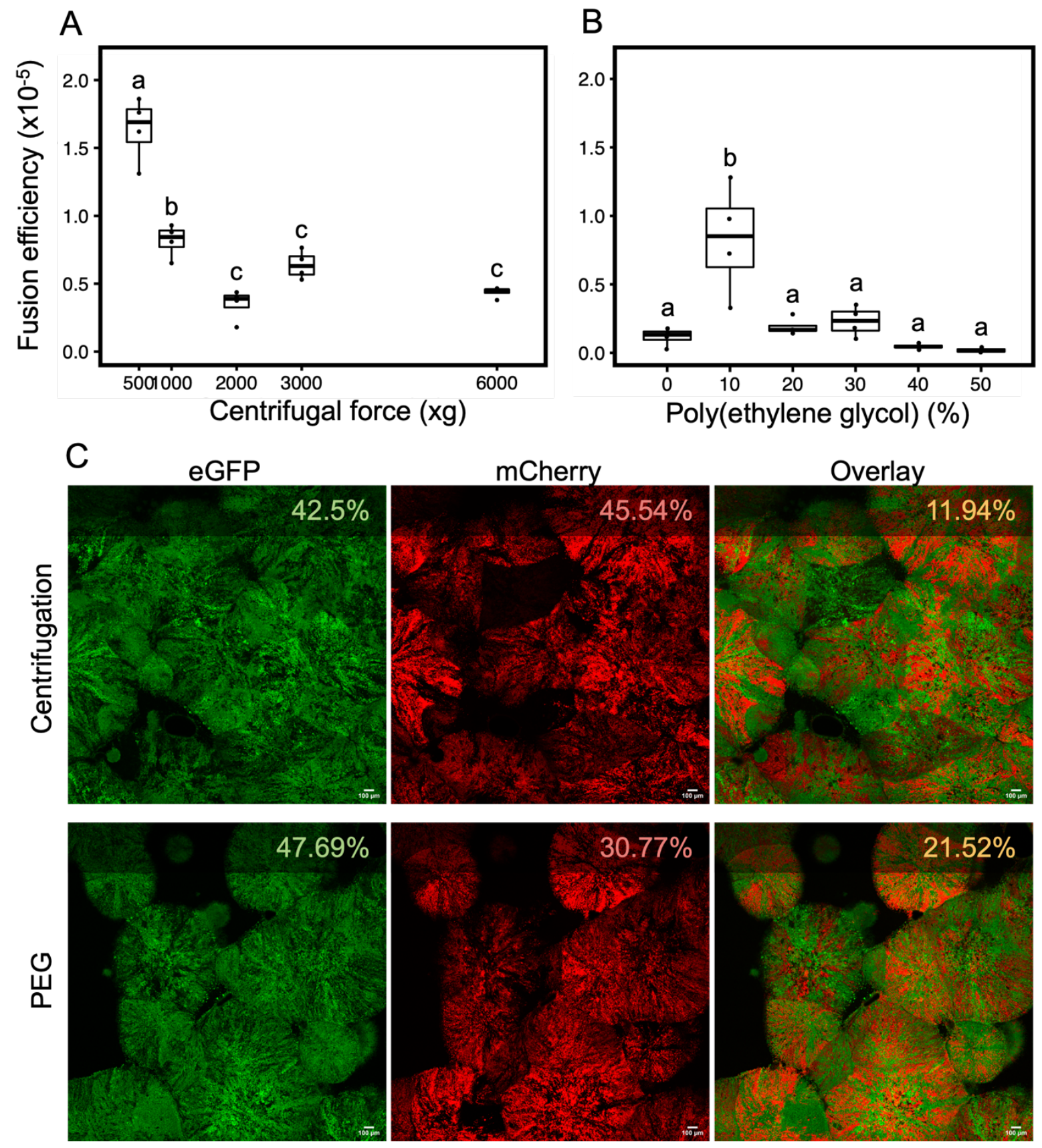

Figure 3. Cell-cell fusion of L-forms. Non specific cell fusion was carried out using either a physical method (centrifugation (A)) or chemical method (poly(ethyleneglycol) (B)). The fusion efficiency was calculated by dividing the total cell count obtained on double selection media with the cell count of individual parent strain (AG or HR). Increasing centrifugal force leads to a decrease in efficiency (oneway ANOVA, $f=15, p=9.77 \times 10^{-9}$, groupwise comparison Tukey's HSD). Poly(ethyleneglycol) concentrations also affected fusion efficiency (one-way ANOVA, $f=22, p=0.033$, groupwise comparison Tukey's HSD) with $10 \%$ w resulting in the highest efficiency of fusion. (C) Fluorescence microscopy of colonies on double antibiotic media after fusion via centrifugation (top panel) and PEG $10 \% \mathrm{w}$ (bottom panel). Fluorescence expression (EGFP and mCherry) is indicated as percent in the top right corner of each image and was calculated using ImageJ/Fiji. The overlay image (third column) shows the percent or area occupied by both green and red pixels and is slightly higher for PEG induced fusion. Scale bar $=100 \mu \mathrm{m}$. 


\section{Fused cells are viable and can proliferate}

174 Successful cell-cell fusion events between different L-form strains combines the

175 cytoplasmic contents and genomes of these cells. To study whether these fused cells

176 (i.e. fusant) are viable, timelapse microscopy of individual cells was performed. In

177 viable growing L-forms, membrane extension and blebbing takes place first along with

178 deformation of cell shape (Mercier, Kawai, and Errington 2013; Studer et al. 2016).

179 This is followed by daughter cell formation which tend to remain attached to the mother

180 cell. Given the non-binary nature of cell division in wall deficient cells it was difficult to

181 track the exact number of daughter cells originating from one mother cell. Using the

182 wildtype L-forms as a reference for cell growth we looked for the same pattern in fused

183 cells which were viable in the presence of both antibiotics. Colonies from a fusion

184 event were inoculated in double selection liquid media to obtain suspended cultures

185 that could be introduced into a 96 well plate for timelapse imaging in an automated

186 microscope. We applied brightfield and fluorescence imaging every 10 min for over a

187 period of 16 hours (Fig. 4). Importantly, the fused L-forms follow the growth

188 characteristics of wild-type/parental strains as evidenced by blebbing and membrane

189 deformation, as well as smaller daughter cells visibly attached to mother cells (Fig. 4,

190 supplementary movie 1). The fusants also show growth upon subculture into fresh

191 medium containing both selection pressures (supplementary Fig. 2). 

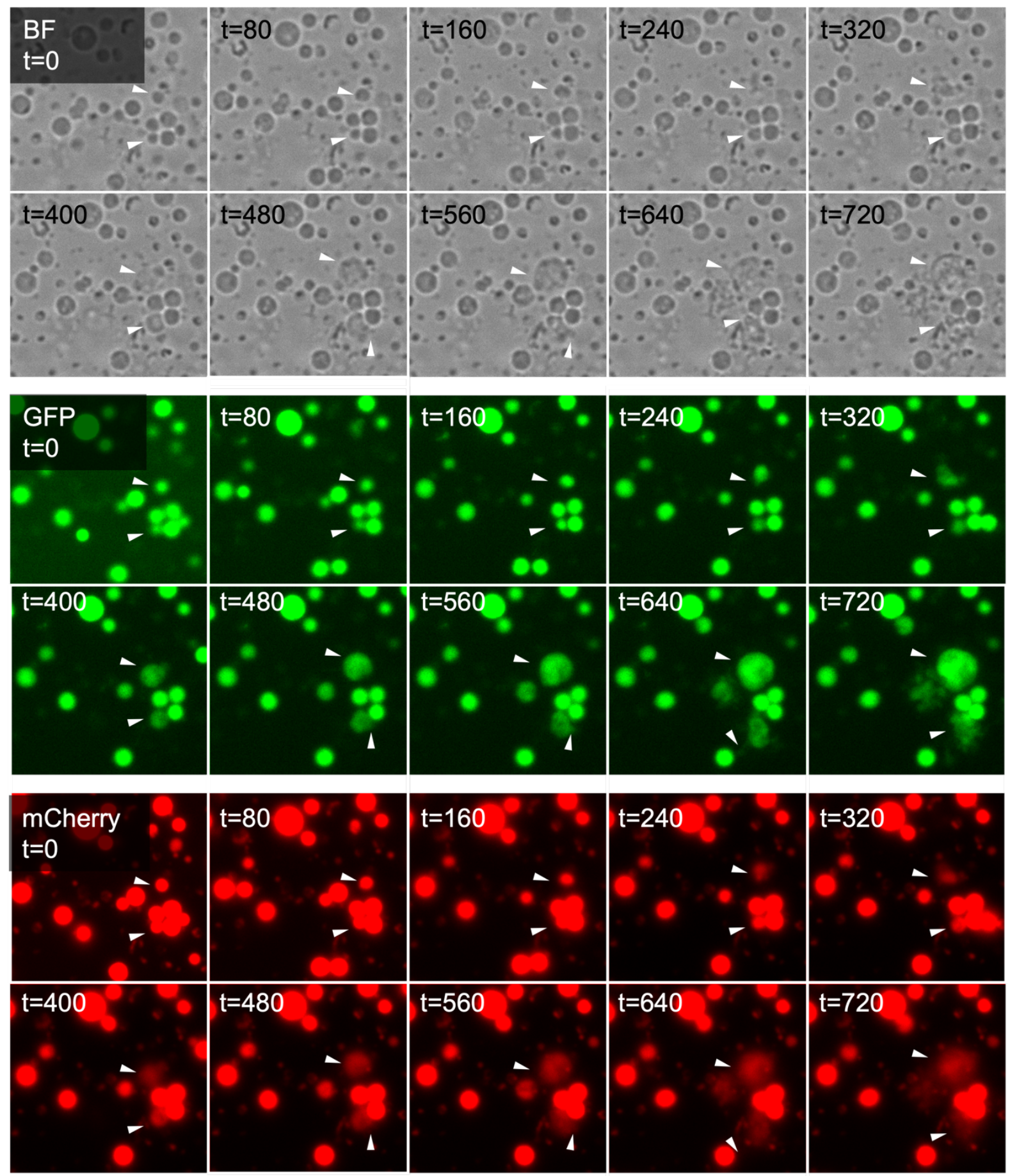

Figure 4. Viability of fused cells. Growth and division of fused cell was tracked over time with brightfield (BF) and fluorescence (GFP and mCherry) microscopy. Images were taken every 10 minutes for a total of 16 hours. The panels (top-BF, middle-GFP, bottom-mCherry) consist of a select few images over this time period (labelled on the top left corner in minutes). White arrows indicate growing cells and membrane extensions. Fused cells also express both fluorescence markers made possible due to cell-cell fusion.

201 The bacterial cell membrane largely consists of (phospho)lipids and fatty acids,

202 together with other minor components. The characteristics of these lipids and fatty 
acids (FA), such as the degree of unsaturation and headgroup composition, determine the physical properties of a membrane. The fluidity of membranes is an important factor governing its fission and fusion ability (Mercier, Domínguez-Cuevas, and Errington 2012; Prives and Shinitzky 1977). Membrane fluidity of L-form cells was quantified as generalized polarization (GP) using the Laurdan dye assay (Scheinpflug, Krylova, and Strahl 2017). This GP value can range from -1 to +1 and inversely correlates to membrane fluidity (i.e., a low GP value indicates a more fluid membrane). Measuring the fluidity for L-forms grown for 1, 3, 5 and 7 days, resulted in a significant

211 GP value increase over time (Fig. $5 A$, rho=0.732, $p=1.87 \times 10^{-6}$ ), indicating that 212 membrane rigidity increases as the cultures age. Importantly, this change in fluidity 213 with culture age negatively correlated with the fusion efficiency, as younger cultures 214 fused at twice the efficiency of older cultures (Fig. 5A inset, unpaired t test, $p=2.22 \times 10^{-}$ $215^{6}$ ). To assess the underlying molecular causes for this shift in fluidity, the membrane 216 lipid and FA composition was analyzed using mass spectrometry from L-form cultures of different ages. Over a 7-day period, there was a significant shift in (phospho)lipid/FA composition as the fraction of saturated FAs increased at the expense of unsaturated FAs (Fig. 5B, top panel). This change is consistent with previous reports in Streptomyces sp. and Bacillus sp. showing that membrane fluidity decreases due to the presence of saturated FAs that stack tightly and thereby make membranes rigid (Mercier, Domínguez-Cuevas, and Errington 2012; Hoischen et al. 1997). In addition, the percent of phosphatidylethanolamine (PE) which is known to affect membrane curvature declines with culture age in L-forms. Both factors, an increase in saturated FAs and a decrease in PE, likely underlie the shift in fusion frequency with colony age, although by different mechanisms.

To causally confirm the impact of membrane fluidity with fusion efficiency, we directly manipulated membrane fluidity by adding PEG into the medium, which is known to induce fusion between two membranes by hydrogen bonding and force adjacent membranes into close proximity via dehydration (MacDonald 1985; Wojcieszyn et al. 1983). When we tested the effect of increasing PEG concentrations on L-form membrane fluidity, we observed a significant positive correlation between GP values and PEG concentrations ( $r h o=0.834, p=1.41 \times 10-6)$ (Fig. 5C) This shows that an increase in PEG leads to reduced membrane fluidity in L-forms. In turn, this caused a decrease in fusion efficiency. Thus a high GP value (i.e. low membrane fluidity) results in low fusion (rho=-0.762, $p=3.74 \times 10^{-5}$ ) (Fig. 5D). 

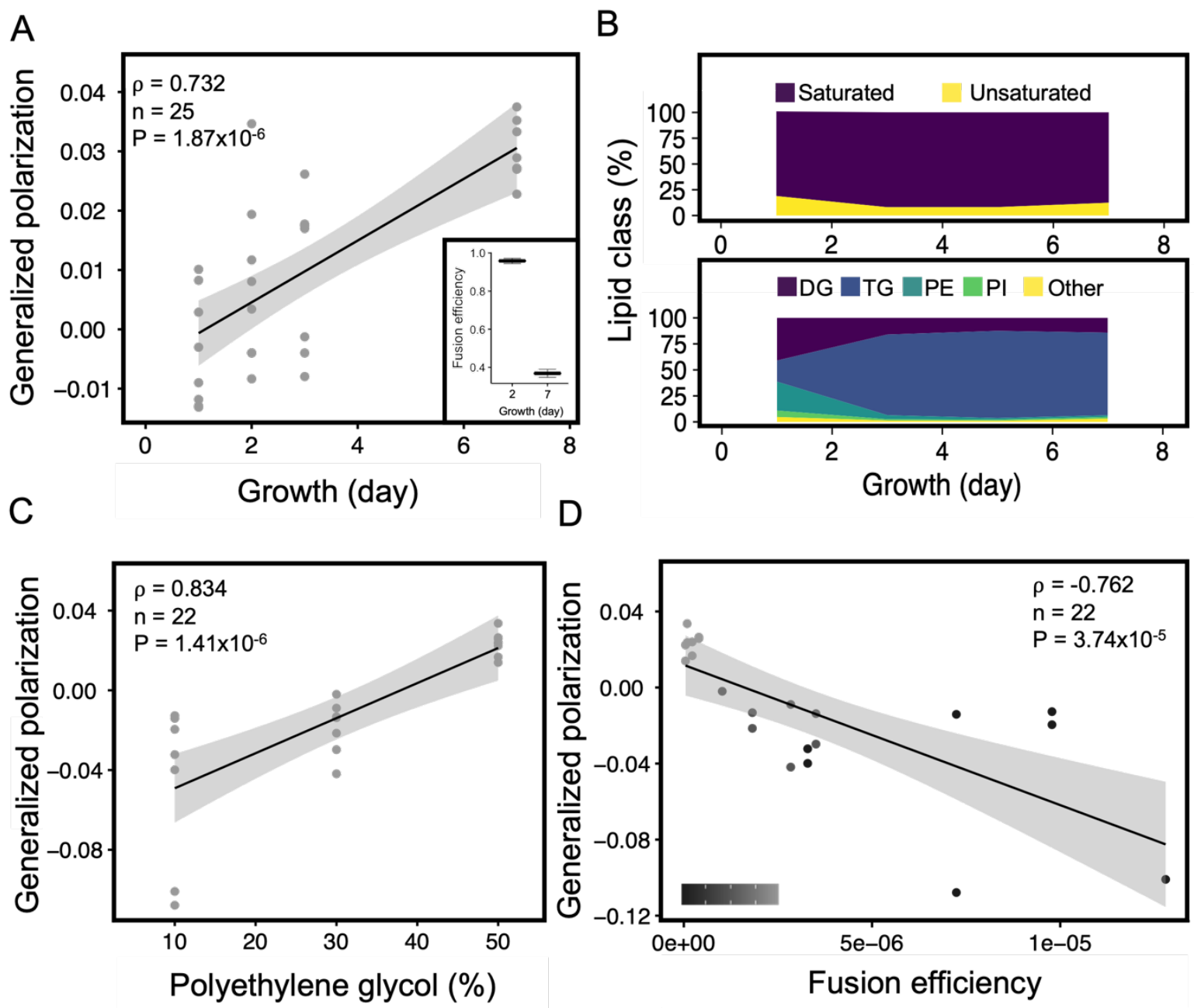

Figure 5. Membrane fluidity affects L-form fusion. (A) Fluidity of L-form membranes was quantified as a generalized polarization (GP) value using the Laurdan dye assay. A strong positive correlation was obtained between GP value and the period of growth indicating a decrease in membrane fluidity with increasing culture age (Spearman's rank correlation test). Age of the culture also has an effect on fusion efficiency (inset, 2 sample $t$ test, $p=2.22 \times 10^{-6}, n=3$ ) with young 2 day old cultures fusing more efficiently than older 7 day old cultures. (B) Analysis of membrane lipids of cultures from different period of growth $(1,3,5$ and 7 day) indicated a change in the percent of saturated and unsaturated fatty acids over time. Specifically the triglyceraldehyde (TG) and phosphatidylethanolamine (PE) show a strong decrease between 1 and 3 day. Both lipids are required for fluidity of the membrane. (C) Positive correlation obtained between GP value and the percent of PEG indicating a decrease in membrane fluidity with increasing concentration of PEG (Spearman's rank correlation test). (D) The GP value shows a strong negative correlation with fusion efficiency. A low percent of PEG (10\%) leads to slightly more fluid membranes compared to a high PEG percent $(50 \%)$ resulting in higher fusion (Spearman's rank correlation test). The grayscale (bottom left corner) indicates PEG percent ranging from 10 to 50 . 
258 Coiled coil lipopeptides localize to L-form membranes and alter membrane

259 fluidity

260 PEG-mediated fusion and centrifugation cause non-specific cell fusion and this can 261 result in a low percent of fused cells expressing both EGFP and mCherry (Fig. 2). The 262 recent use of lipidated peptides in cell fusion has shown great promise to improve 263 fusion efficiency, with examples of successful fusion between liposomes or liposomes 264 with various eukaryotic cell lines (Rabe et al. 2014; Yang, Shimada, et al. 2016; Kong et al. 2020; Yang, Bahreman, et al. 2016). Coiled coil is a common protein structural motif (supplementary Fig. 3) that contains two or more alpha-helices wrapped around each other to form a left-handed superhelical structure (Koukalová et al. 2018; Robson Marsden and Kros 2010). In previous studies, de novo designed coiled coil forming lipopeptides $\mathrm{K}_{4}$ and $\mathrm{E}_{4}$ were conjugated to cholesterol via a flexible PEG-4 spacer, yielding lipopeptides denoted as $\mathrm{CPK}_{4}$ and $\mathrm{CPE}_{4}$ (Versluis et al. 2013; Zope et al. 2013). Using this coiled coil membrane fusion system, efficient liposome-liposome and cell-liposome fusion has been achieved resulting in efficient cytosolic delivery of cargo (Rabe et al. 2014; Yang, Shimada, et al. 2016; Kong et al. 2020). Since L-forms do not posses a cell wall and its outer membrane is structurally similar to (giant) lipid vesicles, we investigated whether coiled-coil lipopeptides $\mathrm{CPE}_{4} / \mathrm{CPK}_{4}$ can be applied to increase the L-form fusion efficiency and introduce cell-specificity. First, we tested whether lipopeptide $\mathrm{CPK}_{4}$ could be inserted in the L-form membrane and still form a coiled coil with its binding partner lipopeptide $\mathrm{E}_{4}$ (Fig. 2, supplementary fig. 3). Incorporating the $\mathrm{CPK}_{4}$ lipopeptide in the membrane allowed docking of the complementary fluorescent labeled peptide $\mathrm{E}_{4}$ (fluo- $\mathrm{E}_{4}$; Fig. 6A). Docking was also observed when $\mathrm{CPE}_{4}$ was incorporated in the L-form membrane, followed by the addition of fluorescent labeled peptide fluo- $\mathrm{K}_{4}$. In contrast, no fluorescence was observed when only fluo- $\mathrm{K}_{4}$ or fluo- $\mathrm{E}_{4}$ was added to L-forms (Fig. 6B). Using image analysis software, we further confirmed membrane localization of the lipopeptidefluorescent dye conjugate by assessing the fluorescence intensity across the cell along a transect line. A combined plot (supplementary fig. 4) of these intensity values across 10 cells indicates coinciding peaks of fluorescence values of the lipopeptide conjugates with that of gray values of the cell membrane (seen as dark grey rings in brightfield images). The fluorescence intensity on L-form membranes was more distinct when $\mathrm{CPE}_{4} /$ fluo- $\mathrm{K}_{4}$ was used as compared to $\mathrm{CPK}_{4} /$ fluo-E 4 (supplementary fig. 4A). Altogether, these results demonstrate for the first time that lipopeptides can 

complementary (lipo)peptides.

A
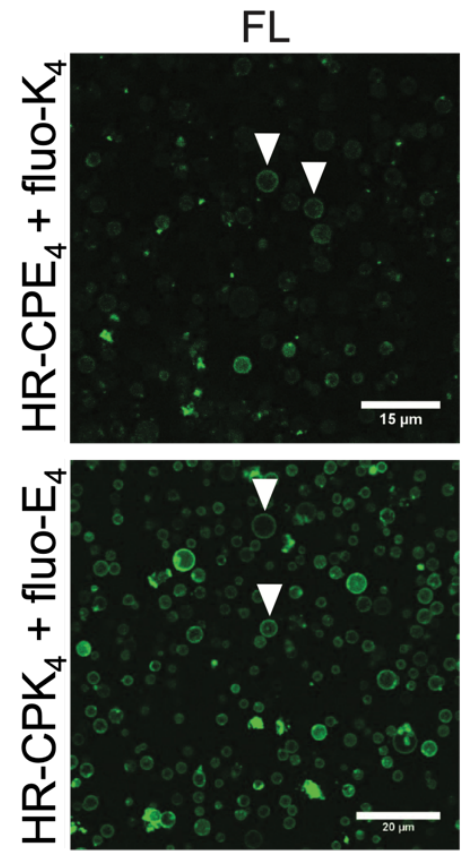

B
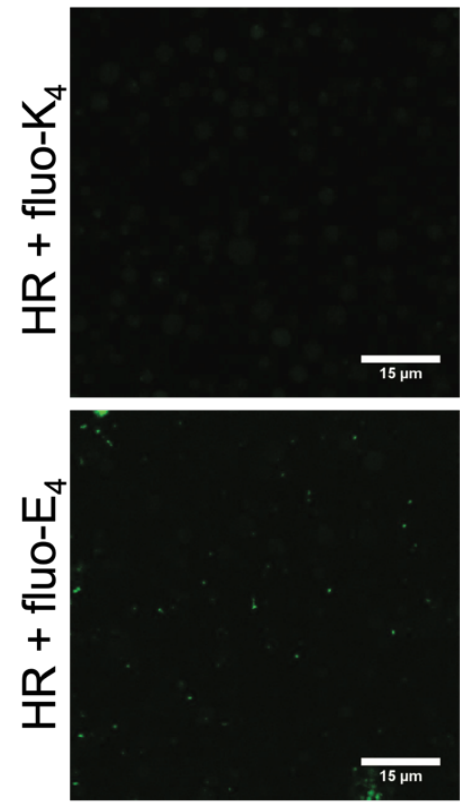

$\mathrm{FL}+\mathrm{DIC}$
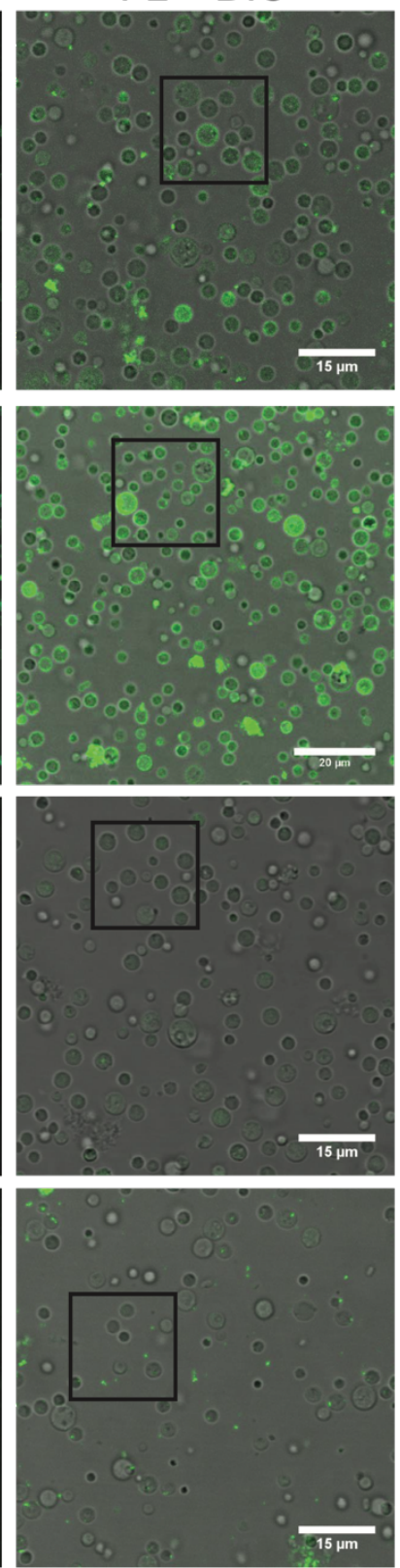

Inset
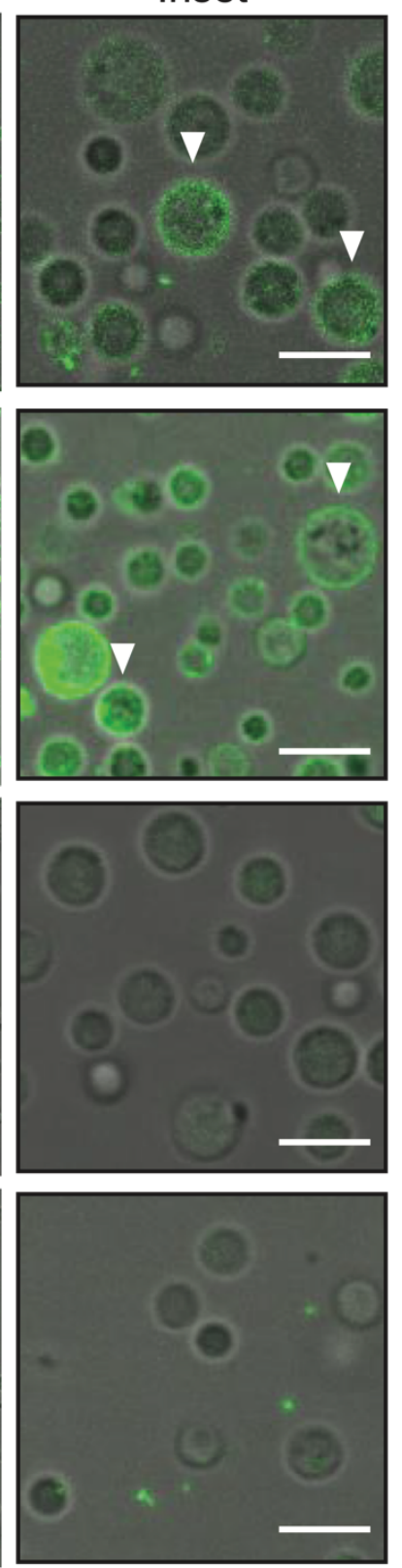

Figure 6. Coiled coil lipopeptides integrate in L-form membranes. (A) Confocal microscopy images (fluorescence $(\mathrm{FL})$ and overlay $(\mathrm{FL}+\mathrm{DIC})$ ) indicating peptide $\mathrm{CPE}_{4}$ or $\mathrm{CPK}_{4}$ insertion into the L-form membranes and coiled-coil formation with complementary peptides (fluo- $K_{4}$ or fluo- $E_{4}$ ). White arrows indicate clear membrane insertion. (B) In the absence of $\mathrm{CPE}_{4}$ or $\mathrm{CPK}_{4}$ no binding of the complementary fluorescent peptides (fluo- $\mathrm{K}_{4}$ or fluo- $\mathrm{E}_{4}$ ) was observed. Experiments were performed at $30^{\circ} \mathrm{C}$, L-forms in P-buffer were incubated with $10 \mu \mathrm{M}$ of $\mathrm{CPE}_{4}$ or $\mathrm{CPK}_{4}$ for 30 minutes. Subsequently the unbound peptide was washed via centrifugation and the complementary fluorescent peptides were added. Scale bar $=5 \mu \mathrm{M}$. 
expressing red fluorescent protein (AR and HR strains) were modified with either nonfluorescent labeled $\mathrm{CPE}_{4}$ and $\mathrm{CPK}_{4}$ so as not to interfere with the emission spectra of the Laurdan dye. The observed GP values reveal that $\mathrm{CPK}_{4}$ and $\mathrm{CPE}_{4}$ affect the fluidity of L-forms differently. While $\mathrm{CPE}_{4}$ decreased fluidity in the AR strain (Fig. 7A), both lipopeptides increased fluidity in the HR strain (Fig. 7B). Interestingly the effect of increased fluidity due to PEG (10 w\%) was only observed in the AR strain. These differences in fluidity effects are likely caused by the presence of antibiotics during culturing of the strains prior to the experiment, which are required to avoid contamination in the cultures (supplementary fig. 5). Antibiotics are known to affect membrane fluidity (Bessa, Ferreira, and Gameiro 2018), however the exact mechanism by which they do so is unclear. This inherent difference was observed in the basal GP values of control samples (-0.02 for HR and -0.08 for AR, Fig. 7A-B) as well as in separate measurements for fluidity of strains in the absence and presence of antibiotics ( 0.01 for $H R$ and -0.10 for $A R$, supplementary fig. 5). However, all treatments (PEG/lipopeptide) are compared to the control sample of individual strain type; hence, the change in GP value is indeed due to the lipopeptide interaction and the to the presence of antibiotics.

We next examined how these changes in fluidity affect the process of lipopeptide-mediated fusion. For this, L-form cultures were first adjusted to the same density and split into aliquots. The aliquots were then either untreated (control), treated with PEG or increasing concentrations of the lipopeptide that previously caused an increase in fluidity. $\mathrm{HR}$ strains were hence pretreated with $\mathrm{CPE}_{4}$ and $\mathrm{AG}$ strains were treated with $\mathrm{CPK}_{4}$. After treatment for 30 minutes the excess PEG and lipopeptides were removed by centrifugation and the L-forms were resuspended in fresh P-buffer containing Dnasel. The cultures were then thoroughly mixed in a 1:1 ratio, incubated for 30 minutes at $30^{\circ} \mathrm{C}$ and subsequently plated on selection media for cell quantification. The observed fusion efficiency for each treatment relative to control revealed that treatment of $\mathrm{HR}$ with $\mathrm{CPE}_{4}$ and $\mathrm{AG}$ with $\mathrm{CPK}_{4}$ results in a high fusion

334 efficiency as compared to 10 w\% PEG or the centrifuged control (Fig. 7C).

335 Furthermore, fusion efficiency was not only dependent on lipopeptide concentration 336 (i.e. decreased fusion at $100 \mu \mathrm{M}$ ) but also on the lipopeptide specificity since AG 337 treated with $\mathrm{CPE}_{4}$ resulted in basal level of fusion similar to the control. Higher 338 lipopeptide concentrations also visibly affected cells, causing lysis (data not shown). 
339 Together these results confirm that cell specific fusion of L-forms can be achieved

340 using fusogenic coiled coil lipopeptides.

341 The two approaches (non-specific via PEG and centrifugation and cell-specific

342 using lipopeptides) used here seem to influence fusion by altering membrane fluidity

343 and bringing membranes together. We then investigated whether combining both

344 fusogens would result in an overall higher fusion efficiency. For this the cells were first

345 treated with the lipopeptides (AG L-forms with $\mathrm{CPK}_{4}$ and $\mathrm{HR}$ L-forms with $\mathrm{CPE}_{4}$ ) and

346 split into two aliquots. The first aliquot was directly subjected to fusion by mixing the

347 cultures in a 1:1 ratio whereas the second aliquot was mixed and treated with PEG.

348 Here the PEG remained in the environment during the process of fusion. Efficiency

349 calculations showed a 3-fold higher relative fusion in the latter (Fig. 7D) indicating that

350 combining lipopeptides and PEG is optimal for cell-cell fusion. The presence of

351 lipopeptides on the cell surface aids in complementary L-form pairing (AG with HR)

352 bringing the opposing membranes in close proximity, which is an important first step

353 in fusion. Additionaly PEG potentially further reduces the space by membrane

354 dehydration thus facilitating fusion events. Colony imaging further confirmed the

355 presence of more double labelled cells in treatment with PEG (supplementary Fig. 6). 


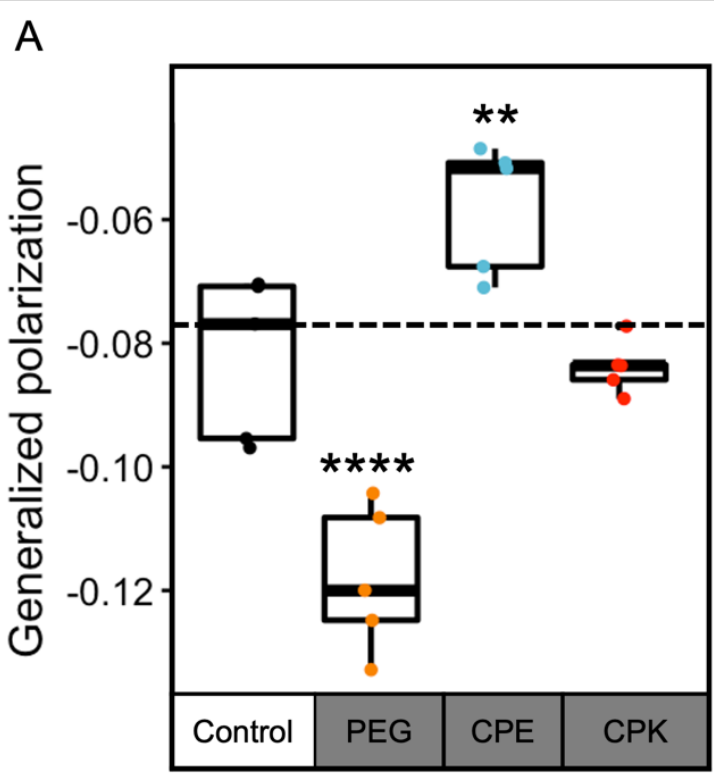

C

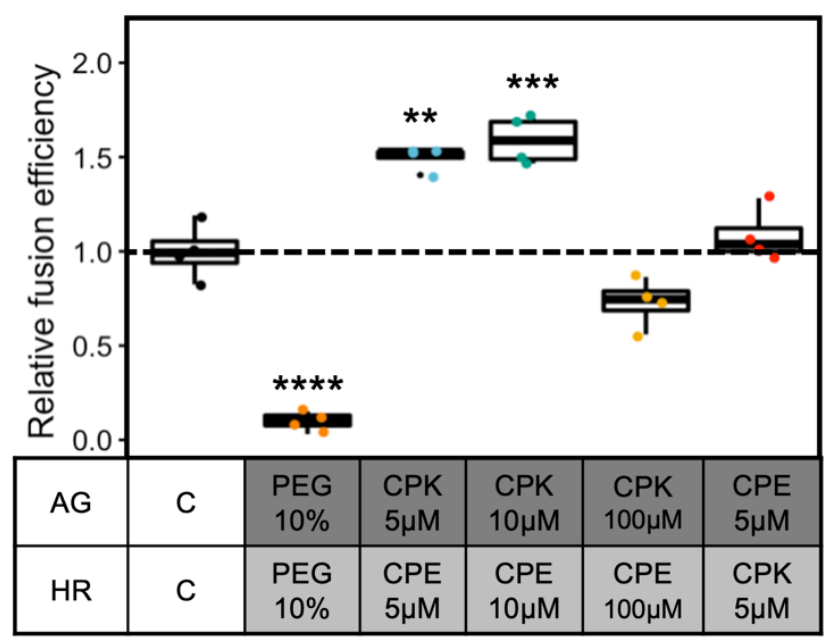

B

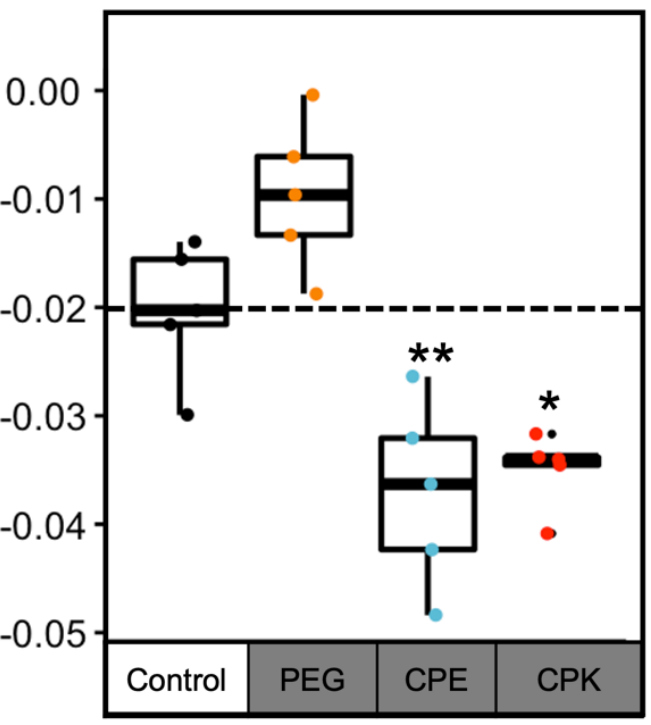

D

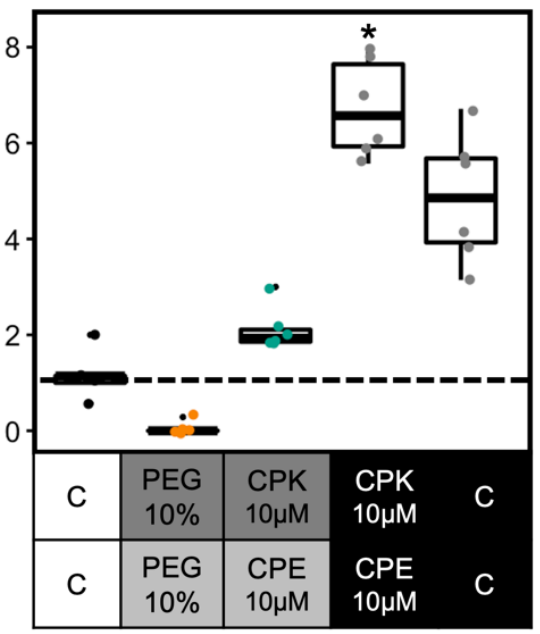

Figure 7. Coiled coil lipopeptides increase membrane fluidity and cell-specific fusion. (A) The strain AR shows an increased fluidity on treatment with PEG $\left(p=3.06 \times 10^{-6}\right)$, a decrease in fluidity on treatment with $\mathrm{CPE}_{4}\left(\mathrm{p}=2.13 \times 10^{-3}\right)$ and no change in fluidity with $\mathrm{CPK}_{4}$ (One-way ANOVA, $\mathrm{F}=36$, $p=4.59 \times 10^{-18}$ followed by Tukey's pairwise comparison) compared to the control (dotted line). (B) The strain HR shows increased fluidity (low GP value) when treated with $\mathrm{CPE}_{4}\left(\mathrm{p}=3.11 \times 10^{-3}\right)$ and $\mathrm{CPK}_{4}$ $\left(p=1.4 \times 10^{-2}\right)$ compared to the control (dotted line) whereas no significant change when treated with $10 \%$ PEG (One-way ANOVA, $F=36, p=2.83 \times 10^{-18}$ followed by Tukey's pairwise comparison). Dotted line is for comparison of GP values to the control where no peptide or PEG was added. (C) The AG and HR strains were individually treated with either PEG, $\mathrm{CPE}_{4}$ or $\mathrm{CPK}_{4}$ at different peptide concentrations to assess the effect on fusion efficiency. Interestingly PEG leads to low fusion despite increasing fluidity because of its non-specific nature. The combination of AG-CPK ${ }_{4}$ and HR-CPE 4 resulted in highest fusion efficiency relative to the basal level. The increase in relative fusion efficiency is concentration dependent as well as peptide dependent (One-way ANOVA, $F=30, p=3.47 \times 10^{-14}$ followed by Tukey's pairwise comparison). (D) The AG and HR strains were first treated with either PEG, CPE 4 or CPK 4 . These strains were then directly plated on double selection media in the absence (grey boxes) or presence (black boxes) of $10 \%$ w PEG to assess the effect on fusion efficiency. Interestingly PEG leads to low fusion despite increasing fluidity because of its non-specific nature when washed away prior to plating but gives a high efficiency when present during the plating. The treatment with peptides also shows a higher efficiency when in the presence of PEG (Kruskall-Wallis chi-squared $=24.84, p=5.4 \times 10^{-}$ ${ }^{5}$, followed by Dunnet's pairwise comparison) compared to the control where no peptide or PEG was added (dotted line). 


\section{Discussion}

379 Cell wall deficiency has primarily been studied in the context of stress tolerance and 380 intracellular pathogenicity (Errington et al. 2016). The genetic and metabolic modifications required to survive in this wall-deficient state are also being uncovered which has deepened our understanding of their intriguing biology (Glover, Yang, and Zhang 2009; Kawai et al. 2019). We here show that wall-deficient L-forms are able to fuse with one another and that membrane fluidity is a key factor influencing fusion efficiency. Additionally, we show for the first time targeted fusion between walldeficient cells using coiled coil lipopeptides. This opens up avenues for application in the field of biotechnology and the design of synthetic cells.

L-forms are surrounded by a membrane, which are be sufficiently fluid to allow efficient proliferation. Bacillus subtilis L-forms that have a defect in formation of branched chain fatty acid (BCFA) suffer from decreased membrane fluidity and as a consequence cannot carry out the membrane scission step (Mercier, DomínguezCuevas, and Errington 2012). This phenotype was rescued by supplementing the media with BCFAs in the medium. Less is known about the impact of fluidity on bacterial fusion, although older reports on eukaryotic muscle cell cultures suggest that myoblast fusion was preceded by a decrease in membrane viscosity (Prives and Shinitzky 1977). In this work we showed that the membrane fluidity of $K$. viridifaciens L-forms changes over time. In younger cultures, the fluidity is higher coinciding with the ability of such cells to proliferate efficiently. By contrast, the fluidity decreases in older cultures. The change in fluidity was associated with a change in the ratio of saturated to unsaturated FAs. In our study we found this ratio to be 4.3 for the $1^{\text {st }}$ day of growth which then increased to 11.3 after 3 days (Fig. 5B). Thus the amount of saturated FAs responsible for tighter packing increases over time at the expense of unsaturated FAs. The accumulation of saturated FAs makes the membrane more stiff, which negatively impacts proliferation and fusion efficiency. Notably, compared to protoplasts, L-forms of Streptomyces hygroscopicus contained 6 times more anteiso FAs than protoplasts resulting in more fluid membranes (Hoischen et al. 1997). Our lipidomics analysis also indicates that L-form membrane composition comprised significant amounts of cardiolipin

(CL), phosphatidylinositol

(PI) and phosphatidylethanolamine (PE). Both CL and PE are fusogenic headgroups shown to induce fusion between liposomes and extracellular vesicles (Driessen et al. 1985), and their the presence may also facilitate L-form fusion. 
A pair of complementary fusogenic coiled coil lipopeptides have been

413 previously developed for the targeted delivery of compounds into eukaryotic cells

414 using liposomes. These eukaryotic-liposome models have also been used extensively

415 to understand the process of cell fusion (Daudey et al. 2017). For the first time we

416 explored targeted fusion with these synthetic lipopeptides between bacterial cells.

417 Interestingly we observed that the lipopeptides readily insert in membranes of L-forms

418 via a cholesterol anchor (Fig. 6). These lipopeptides remained in the membrane even

419 after several washing steps. The lipopeptide segment of $\mathrm{CPK}_{4}$ is known to interact

420 both with its binding partner lipopeptide $\mathrm{E}_{4}$ as well as membranes while the lipopeptide

$421 \mathrm{E}_{4}$ segment of $\mathrm{CPE}_{4}$ does not (Fig. 2). Complementary binding of the lipopeptides

422 brings two opposing membranes in close proximity and ultimately induces fusion

423 (Koukalová et al. 2018; Robson Marsden et al. 2009). The differences in lipopeptide

424 presentation on the surface can explain the complementarity effect on fusion efficiency

425 of L-forms as well (Fig. 7). Given the ease of lipopeptide docking and subsequent

426 stability on the L-forms, coiled coil lipopeptides provide a promising avenue for studies

427 on targeted compound delivery into wall deficient cells. This may be particularly

428 relevant for L-forms associated with recurring urinary tract infections and potentially

429 mycobacterial infections (Mickiewicz et al. 2019; Markova 2017).

430 The costs and benefits of living as a wall deficient cell depends on the

431 environment. Absence of a protective wall makes them sensitive to changes in osmotic

432 pressure and physical agitation. On the other hand, cells without a wall are resistant

433 to a whole class of cell wall targeting antibiotics (penicillins, cephalosporins), transport

434 to the extracellular space is potentially easier and the cells are stably polyploid. These

435 characteristics can make L-forms a unique model system to study not only cell biology

436 but also questions in the fields of biotechnology, evolution and the origin of life (Briers

437 et al. 2012; Errington et al. 2016; Shitut et al. 2020). The process of cell fusion may

438 have been a mechanism of horizontal gene transfer and species diversification in early

439 life (Küppers and Zimmermann 1983). Understanding this process is hence a key

440 aspect of protocell evolution. L-forms are uniquely suited to replicate these processes

441 thereby providing a mechanistic understanding of the causes and consequences of

442 such fusion. First, the use of coiled coil directed fusion can be extended to synthetic

443 cells to obtain fusions that increase cellular complexity. Second, fusion leads to

444 multiple chromosomes in the same cellular compartment which in turn can result in

445 genetic recombination. Such recombination events can then be leveraged to identify 
new microbial products and obtain genomically diverse populations of cells. Finally, cell-cell fusion can also help to understand major transitions on the road to increased organismal complexity like multicellularity and endosymbiosis.

\section{Materials and methods}

Media and growth conditions

All L-form strains were cultured in liquid $L$ phase broth (LPB) and solid $L$ phase media agar (LPMA). LPB consists of a 1:1 mixture of yeast extract malt extract (YEME) and tryptic soy broth supplemented with $10 \%$ sucrose (TSBS) and $25 \mathrm{mM} \mathrm{MgCl} 2$. LPMA consists of LPB supplemented with $1.5 \%$ agar, $5 \%$ horse serum and $25 \mathrm{mM} \mathrm{MgCl} 2$ (Kieser et al. 2000). P-buffer containing sucrose, $\mathrm{K}_{2} \mathrm{SO}_{4}, \mathrm{MgCl}_{2}$, trace elements, $\mathrm{KH}_{2} \mathrm{PO}_{4}, \mathrm{CaCl}_{2}$, TES (Kieser et al. 2000) was used for transformation and all fusion experiments supplemented with $1 \mathrm{mg} / \mathrm{mL}$ Dnasel (Roche Diangnostics $\mathrm{GmbH}$ ). Antibiotics apramycin (Duchefa Biochemie) and hygromycin (Duchefa Biochemie) were used for selection and were added at final concentrations of $50 \mu \mathrm{g} / \mathrm{mL}$ and 100 $\mu \mathrm{g} / \mathrm{mL}$ respectively. Growth conditions for all cultures was $30^{\circ} \mathrm{C}$ in an orbital shaker (New Brunswick Scientific Innova ${ }^{\circledR}$ ) with 100 rpm for the liquid cultures. Centrifugation (Eppendorf Centrifuge 5424) conditions were always $1000 \mathrm{xg}$ for 10 minutes $(<1 \mathrm{~mL})$ or 30 minutes $(>10 \mathrm{~mL})$ depending on culture volume. The above mentioned culture conditions and centrifugation settings were applied throughout the study unless mentioned otherwise. All measurements for optical density of samples was done with $200 \mu \mathrm{L}$ culture in a 96 well flat bottom plate (Sarstedt) using the Tecan spectramax platereader.

\section{Strain and plasmid construction}

Wall deficient L-form of Kitasatospora viridifaciens was obtained by prolonged exposure to penicillin and lysozyme similar to a previous study (Ramijan et al. 2018). Briefly, $10^{6}$ spores of Kitasatopsora viridifaciens DSM40239 were grown in $50 \mathrm{~mL}$ TSBS media at $30^{\circ} \mathrm{C}$ and $100 \mathrm{rpm}$ to obtain mycelial biomass. To this biomass 1 $\mathrm{mg} / \mathrm{mL}$ lysozyme (Sigma Aldrich) and $0.6 \mathrm{mg} / \mathrm{mL}$ penicillin (Duchefa Biochemie) was added to induce S-cell formation. After 7 days, a dense culture of wall-deficient cells was obtained and subcultured to LPB media containing $6 \mathrm{mg} / \mathrm{mL}$ penicillin. This treatment was continued for 5 weeks with subculture into fresh media every week. The 
culture was then tested for growth on LPMA without penicillin and showed only L-form growth. A single colony was picked and inoculated in LPB without penicillin and incubated for 7 days to confirm stability of wall-deficiency and subsequently used for making a culture stock to be stored at $-80^{\circ} \mathrm{C}$.

The strain was further genetically modified to harbour antibiotic resistance genes and fluorescent reporter genes. Two plasmids were used for this purpose namely pGreen (containing the apramycin resistance gene aac(3)IV and a green fluorescent protein reporter gene) and pRed2 (containing the hygromycin resistance gene $h p h$ and a red fluorescent reporter gene). Both plasmids contain the Phi C31 aatP site and a Phi C31 integrase which allows for integration of the marker set at the attB site in the genome. The $\mathrm{pGreen}$ plasmid was obtained from a previous publication where details are provided of the construction (Zacchetti et al. 2016). The pRed2 plasmid was constructed by introducing the amplified mCherry gene alongwith a gap1 promoter region at the Xbal site in the plJ82 plasmid. Briefly, the mCherry gene was amplified together with the gap1 promoter using primers (Sigma) mentioned in supplementary table 1 and the pRed plasmid (Zacchetti et al. 2016) as template. The amplified gap1-mCherry product was purified using a kit following instructions of the supplier (Illustra ${ }^{\mathrm{TM}}$ GFX ${ }^{\mathrm{TM}}$ gel band purification kit). The purified product was introduced into the vector plJ82 at the Xbal site (New England Biolabs $\mathrm{GmbH}$ ). This plasmid was first transformed into E. coli DH5alpha for amplification followed by transformation into E. coli ET12567 for demethylation.

The plasmids were introduced into the L-forms by polyethylene glycol (PEG1000 NBS Biologicals) induced transformation similar to protoplast transformation with some modifications (Kieser et al. 2000). L-form cultures were grown for 4 days. Cultures were centrifuged to remove the spent media and the pellet was resuspended in $1 / 4^{\text {th }}$ volume P-buffer. Approximately $500 \mathrm{ng}$ plasmid was added to the resuspended pellet and mixed thoroughly. PEG1000 was added to this mix at a final concentration of $25 \mathrm{w} \% \mathrm{w}$ and mixed gently. After a brief incubation of 5 minutes on the bench the tube was centrifuged. The supernatant was discarded and the pellet resuspended in LPB medium and incubated for 2 hours. The culture was then centrifuged again and the pellet resuspended in $100 \mu \mathrm{L}$ LPB for plating on LPMA media containing selective antibiotics apramycin or hygromycin. After 4 days of incubation single colonies were picked and restreaked on LPMA with antibiotics for confirmation along with fluorescence microscopy. The resulting strains were named 
514 AG for Apramycin-Green and HR for Hygromycin-Red and will be referred so 515 henceforth.

516 To test the antibiotic susceptibility, both strains were grown on LPMA containing 517 with or without either $50 \mu \mathrm{g} / \mathrm{mL}$ apramycin or $100 \mu \mathrm{g} / \mathrm{mL}$ hygromycin for 4 days. 518 Stepwise 10-fold dilution plating was done which allowed for quantifying the number 519 of colonies (CFU/mL).

\section{L-form fusion}

522 Strains $A G$ and HR were grown individually from culture stocks in $20 \mathrm{~mL}$ LPB containing the relevant antibiotic. Grown cultures were then centrifuged to remove spent media containing antibiotics and washed with P-buffer twice. The pellet was finally resuspended in 2-3 $\mathrm{mL}$ of P-buffer containing DNase I $(1 \mathrm{mg} / \mathrm{mL})$ and the density was adjusted to $0.6 \mathrm{OD}_{600}$. Both strains were then mixed in equal volumes $(200 \mu \mathrm{L})$ in a fresh microfuge tube and mixed gently followed by incubation at room temperature for 10 minutes. Depending on the treatment, PEG1000 was added at the desired concentration ( 0 to $50 \mathrm{w} \%$ ) and mixed by pipetting. For the effect of centrifugation on L-form fusion no PEG was added. After a brief incubation of 5 minutes the tubes were centrifuged and the supernatant was discarded. The pellet was resuspended in $100 \mu \mathrm{L}$ of P-buffer with DNase I and serial dilutions were subsequently plated on LPMA with both antibiotics. Controls were also plated on the same medium such as $100 \mu \mathrm{L}$ monocultures of each strain to test for cross resistance and $100 \mu \mathrm{L}$ of $1: 1 \mathrm{mix}$ of each strain without fusion (supplementary figure 1). All plates were incubated for 3 days after which colony forming units were calculated to determine the fusion efficiency. Effiiciency was quantified as the CFU/mL on double antibiotic selection media normalized by the CFU/mL of monocultures grown on single antibiotic selection media.

Microscopy

A Zeiss LSM 900 airyscan 2 microscope was used to image the fluorescently labeled strains under 40x magnification. For EGFP an excitation wavelength of $488 \mathrm{~nm}$ was used and emission captured at $535 \mathrm{~nm}$ whereas for mCherry an excitation wavelength of $535 \mathrm{~nm}$ was used and emission captured at $650 \mathrm{~nm}$. Multichannel (fluorescence and brighfield), multi-stack images were captured using the Zen software (Zeiss) and further analyzed using ImageJ/Fiji. Multiple tiles were imaged for colonies to cover a 
large area. These tiles were then stitched and each fluorescence channel was first thresholded to determine the total pixel area. These thresholded images were then used to calculate total area (using the OR function in image calculator) and the fused area (using the AND function). The total area selection was then used to calculate individual pixel area occupied by either green or red pixels and by both.

The Lionheart FX automated microscope (BioTek) was used for timelapse imaging of double labeled L-forms after fusion. The fusant strains were precultured in LPB containing both antibiotics for 3 days. These were then centrifuged and resuspended in fresh media with antibiotics and $100 \mu \mathrm{L}$ of this was added to individual wells in a 96 well black/clear bottom sensoplate (Thermoscientific). The plate was centrifuged for 5 minutes to enable settling of cells. The timelapse imaging was done using a 63x dry objective, set for 3 channels (brightfield, green and red) with imagining every 10 minutes for 16 hours at $30^{\circ} \mathrm{C}$. The LED intensity for all channels was 10 and a camera gain of 24 . The exposure time was set at the beginning of the imaging according to the reference monoculture strains $A G$ and $H R$.

\section{Membrane fluidity assay}

565 The membrane fluidity was quantified for cultures of different age and cultures treated with different lipopeptides using the Laurdan dye assay (Scheinpflug, Krylova, and Strahl 2017). All cultures grown in $40 \mathrm{~mL}$ volume were first centrifuged followed by resuspension in P-buffer and density adjusted to 0.6 to $0.8 \mathrm{OD}_{600}$. The cultures were then aliquot according to the treatment for a given biological replicate (i.e. 5 aliquots of $1 \mathrm{~mL}$ each for 5 treatments). In case of lipopeptide treatment the lipopeptide was added to the culture at required concentration $(5 \mu \mathrm{M}, 10 \mu \mathrm{M}$ or $100 \mu \mathrm{M})$ and all tubes were incubated for 30 minutes at $100 \mathrm{rpm}$. Centrifugation was carried out to remove excess lipopeptide and the pellet was resuspended in P-buffer. The P-buffer for this assay was always maintained at $30^{\circ} \mathrm{C}$ so as not to alter fluidity of the membrane. 10 mM Laurdan (6-Dodecanoyl-2-Dimethylaminonapthalene, Invitrogen) stock solution was prepared in $100 \%$ dimethylformamide (DMF, Sigma) and stored at $-20^{\circ} \mathrm{C}$ in an amber tube to protect from light exposure. This stock solution was used to get a final concentration of $10 \mu \mathrm{M}$ in the resuspended cultures above. The tubes were inverted to mix the dye sufficiently and then incubated at $30^{\circ} \mathrm{C}$ for 10 minutes and covered with foil to protect from light exposure. The cultures were then washed $3 x$ in pre-warmed 
unbound dye molecules. The final suspension was done in pre-warmed P-buffer and $200 \mu \mathrm{L}$ was transferred to a 96 well black/clear bottom sensoplate (Thermoscientific) for spectroscopy. Fluorescent intensities were measured by excitation at $350 \mathrm{~nm}$ and two emission wavelengths (435 and $490 \mathrm{~nm}$ ). The background values were first subtracted from all sample values followed by estimation of the generalized polarization (GP) value.

$$
G P=\frac{I_{435}-I_{490}}{I_{435}+I_{490}}
$$

588 The GP value ranges from -1 to +1 with low values corresponding to high membrane 589 fluidity.

\section{Lipid extraction and analysis}

592 Cultures of the wildtype L-form were grown for different time periods $(1,3,5$ and 7 days). These were centrifuged and resuspended in P-buffer prior to membrane lipidomics. Lipids where extracted using a modified MTBE protocol of Matyash, V. et al. (ref. 10.1194/jIr.D700041-JLR200). In short, $600 \mu \mathrm{L}$ MTBE and $150 \mu \mathrm{L}$ methanol were added to the thawed bacteria samples. Samples where briefly vortexed, ultrasonicated for 10 minutes and shaken at room temperature for 30 minutes. Next, 300 $\mu \mathrm{L}$ water was added and the samples where centrifuged for 5 minutes at $18213 \times \mathrm{g}$ at $20{ }^{\circ} \mathrm{C}$. After centrifugation, the upper layer was collected and transferred to a glass vial. The extraction was repeated by adding $300 \mu \mathrm{L}$ MTBE and $100 \mu \mathrm{L}$ methanol. Samples where briefly vortexed and shaken at room temperature for 5 minutes. Next, $100 \mu \mathrm{L}$ water was added and the samples where centrifuged for 5 minutes at 18213 $\times g$ at $20{ }^{\circ} \mathrm{C}$. After centrifugation, the upper layer was collected, and the organic extracts combined. Samples where dried under a gentle stream of nitrogen. After drying samples were reconstituted in $100 \mu \mathrm{L}$ 2-propanol. After briefly vortexing and ultra-sonication for 5 minutes, $100 \mu \mathrm{L}$ water was added. Samples were transferred to microvial inserts for analysis based lipid profiling method (PMID: 31972163 DOI: 10.1016/j.bbamem.2020.183200). A Shimadzu Nexera X2 (consisting of two LC30AD pumps, a SIL30AC autosampler,

611 a CTO20AC column oven and a CBM20A controller) (Shimadzu, 's Hertogenbosch, 612 The Netherlands) was used to deliver a gradient of water:acetonitrile 80:20 (eluent A) 613 and water:2-propanol:acetonitrile 1:90:9 (eluent B). Both eluents contained $5 \mathrm{mM}$ 
614 ammonium formate and $0.05 \%$ formic acid. The applied gradient, with a column flow

615 of $300 \mu \mathrm{L} / \mathrm{min}$, was as follows: $0 \mathrm{~min} 40 \% \mathrm{~B}, 10 \mathrm{~min} 100 \% \mathrm{~B}, 12 \mathrm{~min} 100 \% \mathrm{~B}$. A

616 Phenomenex Kinetex C18, $2.7 \mu \mathrm{m}$ particles, $50 \times 2.1 \mathrm{~mm}$ (Phenomenex, Utrecht, The

617 Netherlands) was used as column with a Phenomenex SecurityGuard Ultra C8, 2.7

$618 \mu \mathrm{m}, 5 \times 2.1 \mathrm{~mm}$ cartridge (Phenomenex, Utrecht, The Netherlands) as guard column.

619 The column was kept at $50{ }^{\circ} \mathrm{C}$. The injection volume was $10 \mu \mathrm{L}$.

The MS was a Sciex TripleTOF 6600 (AB Sciex Netherlands B.V., Nieuwerkerk aan den ljssel, The Netherlands) operated in positive (ESI+) and negative (ESI-) ESI mode, with the following conditions: ion source gas 145 psi, ion source gas 250 psi, curtain gas $35 \mathrm{psi}$, temperature $350^{\circ} \mathrm{C}$, acquisition range $\mathrm{m} / \mathrm{z} 100-1800$, ion spray Voltage $5500 \mathrm{~V}(\mathrm{ESI}+)$ and $-4500 \mathrm{~V}(\mathrm{ESI}-)$, declustering potential $80 \mathrm{~V}(\mathrm{ESI}+)$ and -80 $V(E S I-)$. An information dependent acquisition (IDA) method was used to identify lipids, with the following conditions for MS analysis: collision energy \pm 10 , acquisition time $250 \mathrm{~ms}$ and for MS/MS analysis: collision energy \pm 45 , collision energy spread 25 , ion release delay 30 , ion release width 14, acquisition time $40 \mathrm{~ms}$. The IDA switching criteria were set as follows: for ions greater than $\mathrm{m} / \mathrm{z} 300$, which exceed $200 \mathrm{cps}$, exclude former target for $2 \mathrm{~s}$, exclude isotopes within $1.5 \mathrm{Da}$, max. candidate ions 20 . Before data analysis, raw MS data files were converted with the Reifycs Abf Converter (v1.1) to the Abf file format. MS-DIAL (v4.20), with the FiehnO (VS68) database was used to align the data and identify the different lipids (Tsugawa et al. 2015; 2019; 2020). Further processing of the data was done with $R$ version 4.0.2 (R Core Team 2014).

The relative abundance of specific lipid class vs total relative abundance was used to roughly compare the ratio of each lipid class. The lipids have been sorted into saturated and unsaturated lipids classes. Also, the lipids have been sorted based on head groups (DG, TG, PE, PI) and the ratio of each class have been calculated

642 Peptide $\mathrm{K}_{4}$ and $\mathrm{E}_{4}$ were synthesized on a CEM Liberty Blue microwave-assisted 643 peptide synthesizer using Fmoc chemistry. 20\% piperidine in DMF was used as the 644 deprotection agent. During coupling, DIC was applied as the activator and Oxyma as 645 the base. All peptides were synthesized on a Tentagel S RAM resin $(0.22 \mathrm{mmol} / \mathrm{g})$. 646 The resin was swelling for at least 15min before synthesis started. For the coupling, 5 
equivalents of amino acids (2.5 mL in DMF), DIC (1 mL in DMF) and Oxyma (0.5 mL in DMF) were added to the resin in the reaction vessel and were heated to $90{ }^{\circ} \mathrm{C}$ for 4 minutes to facilitate the reaction. For deprotection, $20 \%$ of piperidine (4 $\mathrm{mL}$ in DMF) was used and heated to $90^{\circ} \mathrm{C}$ for 1 minute. Between deprotection and peptide coupling, the resin has been washed three times using DMF. After peptide synthesis, a polyethyleneglycol $(\mathrm{PEG})_{4}$ linker and cholesterol were coupled manually to the peptide on-resin. $0.1 \mathrm{mmol}$ of each peptide was reacted with $0.2 \mathrm{mmol} \mathrm{N} 3-\mathrm{PEG}_{4}-$ $\mathrm{COOH}$ by adding $0.4 \mathrm{mmol} \mathrm{HCTU}$ and $0.6 \mathrm{mmol}$ DIPEA in $3 \mathrm{~mL}$ DMF. The reaction was performed at room temperature for 5 hours. After thorough washing, $3 \mathrm{~mL}$ of 0.5 mmol trimethylphosphine in a 1,4-dioxane: $\mathrm{H}_{2} \mathrm{O}(6: 1)$ mixture was added to the resin to reduce the azide group to an amine (overnight reaction). After reduction, the peptide was reacted with cholesteryl hemisuccinate $(0.3 \mathrm{mmol})$ in DMF by adding $0.4 \mathrm{mmol}$ HCTU and 0.6 mmol DIPEA. The reaction was performed at room temperature for 3 hours. Lipopeptides were cleaved from the resin using $3 \mathrm{~mL}$ of a TFA:triisopropylsilane $(97.5: 2.5 \%)$ mixture and shaking for $50 \mathrm{~min}$. After cleavage, the crude lipopeptides were precipitated by pouring into $45 \mathrm{~mL}$ of $-20{ }^{\circ} \mathrm{C}$ diethyl ether:n-hexane (1:1) and isolated by centrifugation. The pellet of the lipopeptides was redissolved by adding 20 $\mathrm{mL} \mathrm{H}_{2} \mathrm{O}$ containing $10 \%$ acetonitrile and freeze-dried to yield a white powder. Lipopeptides were purified with reversed-phase HPLC on a Shimazu system with two LC-8A pumps and an SPD-20A UV-Vis detector, equipped with a Vydac C4 column (22 $\mathrm{mm}$ diameter, $250 \mathrm{~mm}$ length, $10 \mu \mathrm{m}$ particle size). CPK4 was purified using a linear gradient from 20 to $65 \%$ acetonitrile in water (with $0.1 \%$ TFA) with a $12 \mathrm{~mL} / \mathrm{min}$ flow rate over 36 mins. CPE4 was purified using a linear gradient from 20 to $75 \%$ acetonitrile in water (with $0.1 \%$ TFA) with a $12 \mathrm{~mL} / \mathrm{min}$ flow rate over 36 mins. After HPLC purification, all peptides were lyophilized and yielded white powders.

672 For the fluo- $\mathrm{K}_{4}$ and fluo- $\mathrm{E}_{4}$ synthesis, two additional glycine residues were coupled to 673 the $\mathrm{N}$-terminus of the peptides on resin, before the dye was manually coupled by 674 adding $3 \mathrm{~mL}$ DMF containing $0.2 \mathrm{mmol}$ 5(6)-carboxyfluorescein, $0.4 \mathrm{mmol} \mathrm{HCTU}$ and $6750.6 \mathrm{mmol}$ DIPEA. The reaction was left at room temperature overnight. The fluo- $\mathrm{K}_{4}$ and fluo- $\mathrm{E}_{4}$ were cleaved from the resin using $3 \mathrm{~mL}$ of a TFA:triisopropylsilane: $\mathrm{H}_{2} \mathrm{O}$ $(97.5: 2.5 \%)$ mixture and shaking for 1.5 hours. After cleavage, the crude lipopeptides were precipitated by pouring into $45 \mathrm{~mL}$ of $-20{ }^{\circ} \mathrm{C}$ diethyl ether and isolated by centrifugation. The pellet of the lipopeptides was redissolved by adding $20 \mathrm{~mL} \mathrm{H}_{2} \mathrm{O}$ 
containing $10 \%$ acetonitrile and freeze-dried to yield a white powder. Fluo- $\mathrm{K}_{4}$ and fluo-

$681 \mathrm{E}_{4}$ were purified using the same HPLC described above equipped with a Kinetix Evo

682 C18 column (21.2 mm diameter, $150 \mathrm{~mm}$ length, $5 \mu \mathrm{m}$ particle size). For the fluo-K4,

683 a linear gradient from 20 to $45 \%$ acetonitrile in water (with $0.1 \%$ TFA) with a $12 \mathrm{~mL} / \mathrm{min}$

684 flow rate over 28 mins was used. For fluo-E4, linear gradient from 20 to 55\% was used.

685 After HPLC purification, all peptides were lyophilized and yielded orange powders.

686 The purity of all peptides were determined by LC-MS (supplementary table 2). The

687 structure of all peptides used in this study can be found in supplementary figure 3.

688 Treatment of cultures with different peptides was done by adding externally to cells 689 suspended in P-buffer and incubating for 30 minutes at $30^{\circ} \mathrm{C} 100 \mathrm{rpm}$. Excess peptide

690 was washed by centrifugation.

691

692 L-form membrane labelling

$6933 \times 10^{8}$ wild type L-forms were suspended in $1 \mathrm{~mL}$ of P-buffer. $10 \mu \mathrm{L}$ of $\mathrm{CPK}_{4}$ or $\mathrm{CPE}_{4}$

694 (10 mM in DMSO) was added to the L-form suspension to a final concentration of 100

$695 \mu \mathrm{M}$. After $30 \mathrm{~min}$ incubation at $30^{\circ} \mathrm{C}$ with shaking at $100 \mathrm{rpm}$, the L-forms were washed

696 two times by centrifugation using P-buffer. The L-forms were then suspended in 900

$697 \mu \mathrm{L}$ P-buffer and $100 \mu \mathrm{L}$ of fluo-K $\mathrm{K}_{4}$ or fluo- $\mathrm{E}_{4}(200 \mu \mathrm{M}$ in P-buffer) was added to a final

698 concentration of $20 \mu \mathrm{M}$. After 5 min incubation, the L-forms were washed three times

699 using P-buffer to get rid of the free fluorescent lipopeptides. For control experiments,

700 fluo-K $\mathrm{K}_{4}$ or fluo- $\mathrm{E}_{4}$ were added to non-lipopeptide modified L-form and incubated for 5

701 min. L-form imaging was performed on a Leica SP8 confocal microscopy. Excitation:

$702488 \mathrm{~nm}$, emission: $500-550 \mathrm{~nm}$.

703

704 Peptide induced L-form fusion

705 Strains $A G$ and HR were grown individually from culture stocks in $20 \mathrm{~mL}$ LPB 706 containing the relevant antibiotic. Grown cultures were then centrifuged to remove 707 spent media containing antibiotics and washed with P-buffer twice. The pellet was 708 finally resuspended in 2-3 mL of P-buffer containing DNase I (1 mg mL-1) and the 709 density was adjusted to $0.6 \mathrm{OD}_{600}$. Peptides were added at required concentrations to $7101 \mathrm{ml}$ cultutres of individual strains AG and HR. Cultures were then incubated for 30 711 minutes at $30^{\circ} \mathrm{C}$ with shaking at $100 \mathrm{rpm}$. Excess and unbound peptide was removed 712 via centrifugation and resuspension of pellet in $1 \mathrm{ml} \mathrm{P}$ buffer containing DNase I. Both 713 strains were then mixed in equal volumes $(200 \mu \mathrm{L})$ in a fresh microfuge tube and mixed 
gently followed by incubation at room temperature for 10 minutes. Depending on the treatment cultures were centrifuged followed by treatment with PEG1000 or simply centrifuged. The pellet was resuspended in $100 \mu \mathrm{L}$ of P-buffer with DNase I and serial dilutions were subsequently plated on LPMA with both antibiotics. Controls were also plated on the same medium such as $100 \mu \mathrm{L}$ monocultures of each strain to test for cross resistance and $100 \mu \mathrm{L}$ of $1: 1 \mathrm{mix}$ of each strain without fusion (supplementary figure 1). All plates were incubated for 3 days after which colony forming units were calculated to determine the fusion efficiency. Effiiciency was quantified as the CFU/mL on double antibiotic selection media normalized by the CFU/mL of monocultures grown on single antibiotic selection media.

\section{Statistical analysis and graphs}

Statistical analysis of all datasets was done in R version 3.6.1 (R Core Team 2014) using built-in packages. The specific tests performed are mentioned in the results and figure legends. All graphs were produced using the package ggplot2 (Wickham, H. 2009).

Acknowledgements

We thank members of the Claessen lab and Kros lab for fruitful discussions and suggestions. S.S. acknowledges the NWA startimpulse (Origins Centre) for funding.

Author contribution

S.S., D.C, and A.K. designed the project. S.S. performed all experiments. S.S. and M.S. performed peptide fusion experiments. M.S. prepared all lipopeptides and did microscopy for lipopeptide docking experiments. B.C. prepared the cell-wall-deficient line of K. viridifaciens used in the study. R.D. and M.G. performed the membrane lipid analysis. S.S., D.R., D.C. and A.K. acquired funding. S.S. wrote the first draft followed by revisions from all authors. All authors approved the final manuscript. 
References

Adamala, Katarzyna, and Pier Luigi Luisi. 2011. "Experimental Systems to Explore Division." Cell Cycle in Development, 1-9.

Baltz, Richard H., and Patti Matsushima. 1981. "Protoplast Fusion in Streptomyces: Conditions for Efficient Genetic Recombination and Cell Regeneration." Microbiology, . Microbiology Society,.

Bessa, Lucinda J., Mariana Ferreira, and Paula Gameiro. 2018. "Evaluation of Membrane Fluidity of Multidrug-Resistant Isolates of Escherichia Coli and Staphylococcus Aureus in Presence and Absence of Antibiotics." Journal of Photochemistry and Photobiology B: Biology 181 (April): 150-56.

Briers, Yves, Peter Walde, Markus Schuppler, and Martin J. Loessner. 2012. "How Did Bacterial Ancestors Reproduce? Lessons from L-Form Cells and Giant Lipid Vesicles." BioEssays 34 (12): 1078-84.

Chen, Elizabeth H., Eric Grote, William Mohler, and Agnès Vignery. 2007. "Cell-Cell Fusion." Membrane Trafficking 581 (11): 2181-93.

Chernomordik, L., M. M. Kozlov, and J. Zimmerberg. 1995. "Lipids in Biological Membrane Fusion." The Journal of Membrane Biology 146 (1): 1-14.

Claessen, Dennis, and Jeff Errington. 2019. "Cell Wall Deficiency as a Coping Strategy for Stress." Trends in Microbiology 27 (12): 1025-33.

Daudey, Geert A., Harshal R. Zope, Jens Voskuhl, Alexander Kros, and Aimee L. Boyle. 2017. "Membrane-Fusogen Distance Is Critical for Efficient Coiled-CoilPeptide-Mediated Liposome Fusion." Langmuir 33 (43): 12443-52.

Driessen, A J, D Hoekstra, G Scherphof, R D Kalicharan, and J Wilschut. 1985. "Low $\mathrm{PH}-$ Induced Fusion of Liposomes with Membrane Vesicles Derived from Bacillus Subtilis." Journal of Biological Chemistry 260 (19): 10880-87.

Errington, Jeff, Katarzyna Mickiewicz, Yoshikazu Kawai, and Ling Juan Wu. 2016. "L-Form Bacteria, Chronic Diseases and the Origins of Life." Philosophical Transactions of the Royal Society B: Biological Sciences 371 (1707): 20150494

Glover, William A., Yanqin Yang, and Ying Zhang. 2009. "Insights into the Molecular Basis of L-Form Formation and Survival in Escherichia Coli." PLOS ONE 4 (10): e7316.

Gokhale, D.V., U.S. Puntambekar, and D.N. Deobagkar. 1993. "Protoplast Fusion: A Tool for Intergeneric Gene Transfer in Bacteria." Biotechnology Advances 11 (2): 199-217.

Hanczyc, Martin M, and Jack W Szostak. 2004. "Replicating Vesicles as Models of Primitive Cell Growth and Division." Current Opinion in Chemical Biology 8 (6): 660-64.

Hanson, Phyllis I, John E Heuser, and Reinhard Jahn. 1997. "Neurotransmitter Release - Four Years of SNARE Complexes." Current Opinion in Neurobiology 7 (3): 310-15.

Hoischen, C, K Gura, C Luge, and J Gumpert. 1997. "Lipid and Fatty Acid Composition of Cytoplasmic Membranes from Streptomyces Hygroscopicus and Its Stable Protoplast-Type L Form." Journal of Bacteriology 179 (11): 3430.

Innes, C.M.J., and E.J. Allan. 2001. "Induction, Growth and Antibiotic Production of Streptomyces Viridifaciens L-Form Bacteria." Journal of Applied Microbiology 90 (3): 301-8. 
Kawai, Yoshikazu, Romain Mercier, Katarzyna Mickiewicz, Agnese Serafini, Luiz Pedro Sório de Carvalho, and Jeff Errington. 2019. "Crucial Role for Central Carbon Metabolism in the Bacterial L-Form Switch and Killing by $\beta$-Lactam Antibiotics." Nature Microbiology 4 (10): 1716-26.

Kieser, Tobias, Mervyn J. Bibb, Mark J. Buttner, Keith F. Chater, and David A. Hopwood. 2000. Practical Streptomyces Genetics. Vol. 291. John Innes Foundation Norwich.

Kong, Li, Quanchi Chen, Frederick Campbell, Ewa Snaar-Jagalska, and Alexander Kros. 2020. "Light-Triggered Cancer Cell Specific Targeting and Liposomal Drug Delivery in a Zebrafish Xenograft Model." Advanced Healthcare Materials 9 (6): 1901489.

Kotnik, Tadej. 2013. "Lightning-Triggered Electroporation and Electrofusion as Possible Contributors to Natural Horizontal Gene Transfer." Physics of Life Reviews 10 (3): 351-70.

Koukalová, Alena, Šárka Pokorná, Aimee L. Boyle, Nestor Lopez Mora, Alexander Kros, Martin Hof, and Radek Šachl. 2018. "Distinct Roles of SNAREMimicking Lipopeptides during Initial Steps of Membrane Fusion." Nanoscale 10 (40): 19064-73.

Küppers, G., and U. Zimmermann. 1983. "Cell Fusion by Spark Discharge and Its Relevance for Evolutionary Processes." FEBS Letters 164 (2): 323-29.

MacDonald, Ruby I. 1985. "Membrane Fusion Due to Dehydration by Polyethylene Glycol, Dextran, or Sucrose." Biochemistry 24 (15): 4058-66.

Markova, Nadya. 2017. "L-Form Bacteria Cohabitants in Human Blood: Significance for Health and Diseases." Discovery Medicine 23 (128): 305-13.

Marrink, Siewert J., and Alan E. Mark. 2003. "The Mechanism of Vesicle Fusion as Revealed by Molecular Dynamics Simulations." Journal of the American Chemical Society 125 (37): 11144-45.

Mercier, Romain, Patricia Domínguez-Cuevas, and Jeff Errington. 2012. "Crucial Role for Membrane Fluidity in Proliferation of Primitive Cells." Cell Reports 1 (5): 417-23.

Mercier, Romain, Yoshikazu Kawai, and Jeff Errington. 2013. "Excess Membrane Synthesis Drives a Primitive Mode of Cell Proliferation." Cell 152 (5): 9971007.

Mercier, Romain, Yoshikazu Kawai, and Jeff Errington. 2014. "General Principles for the Formation and Proliferation of a Wall-Free (L-Form) State in Bacteria." ELife 3 (October): e04629.

Mickiewicz, Katarzyna M, Yoshikazu Kawai, Lauren Drage, Margarida C Gomes, Frances Davison, Robert Pickard, Judith Hall, Serge Mostowy, Phillip D Aldridge, and Jeff Errington. 2019. "Possible Role of L-Form Switching in Recurrent Urinary Tract Infection." Nature Communications 10 (1): 4379_ 4379.

Monnard, Pierre-Alain, and David W. Deamer. 2002. "Membrane Self-Assembly Processes: Steps toward the First Cellular Life." The Anatomical Record 268 (3): 196-207.

Naor, Adit, and Uri Gophna. 2013. "Cell Fusion and Hybrids in Archaea." Bioengineered 4 (3): 126-29.

Ogle, Brenda M., Marilia Cascalho, and Jeffrey L. Platt. 2005. "Biological Implications of Cell Fusion." Nature Reviews Molecular Cell Biology 6 (7): 567-75. 
Prives, J., and M. Shinitzky. 1977. "Increased Membrane Fluidity Precedes Fusion of Muscle Cells." Nature 268 (5622): 761-63.

R Core Team. 2014. R: A Language and Environment for Statistical Computing. Vienna, Austria.: R Foundation for Statistical Computing.

Rabe, Martin, Christian Schwieger, Harshal R. Zope, Frank Versluis, and Alexander Kros. 2014. "Membrane Interactions of Fusogenic Coiled-Coil Peptides: Implications for Lipopeptide Mediated Vesicle Fusion." Langmuir 30 (26): 7724-35.

Ramijan, Karina, Eveline Ultee, Joost Willemse, Zheren Zhang, Joeri A. J. Wondergem, Anne van der Meij, Doris Heinrich, Ariane Briegel, Gilles P. van Wezel, and Dennis Claessen. 2018. "Stress-Induced Formation of Cell WallDeficient Cells in Filamentous Actinomycetes." Nature Communications 9 (1): 5164.

Robson Marsden, Hana, Nina A. Elbers, Paul HH Bomans, Nico AJM Sommerdijk, and Alexander Kros. 2009. "A Reduced SNARE Model for Membrane Fusion." Angewandte Chemie 121 (13): 2366-69.

Robson Marsden, Hana, and Alexander Kros. 2010. "Self-Assembly of Coiled Coils in Synthetic Biology: Inspiration and Progress." Angewandte Chemie International Edition 49 (17): 2988-3005.

Scheinpflug, Kathi, Oxana Krylova, and Henrik Strahl. 2017. "Measurement of Cell Membrane Fluidity by Laurdan GP: Fluorescence Spectroscopy and Microscopy." In Antibiotics: Methods and Protocols, edited by Peter Sass, 159-74. New York, NY: Springer New York.

Shitut, Shraddha, Güniz Ö. Bergman, Alexander Kros, Daniel E. Rozen, and Dennis Claessen. 2020. "Use of Permanent Wall-Deficient Cells as a System for the Discovery of New-to-Nature Metabolites." Microorganisms 8 (12).

Smeijers, A. F., A. J. Markvoort, K. Pieterse, and P. A. J. Hilbers. 2006. "A Detailed Look at Vesicle Fusion." The Journal of Physical Chemistry B 110 (26): 13212-19.

Soucy, Shannon M., Jinling Huang, and Johann Peter Gogarten. 2015. "Horizontal Gene Transfer: Building the Web of Life." Nature Reviews Genetics 16 (8): 472-82.

Studer, Patrick, Titu Staubli, Noémi Wieser, Patrick Wolf, Markus Schuppler, and Martin J Loessner. 2016. "Proliferation of Listeria Monocytogenes L-Form Cells by Formation of Internal and External Vesicles." Nature Communications 7 (November): 13631-13631.

Svetina, Saša. 2009. "Vesicle Budding and the Origin of Cellular Life." ChemPhysChem 10 (16): 2769-76.

Szostak, Jack W., David P. Bartel, and P. Luigi Luisi. 2001. "Synthesizing Life." Nature 409 (6818): 387-90.

Tsugawa, Hiroshi, Tomas Cajka, Tobias Kind, Yan Ma, Brendan Higgins, Kazutaka Ikeda, Mitsuhiro Kanazawa, Jean VanderGheynst, Oliver Fiehn, and Masanori Arita. 2015. "MS-DIAL: Data-Independent MS/MS Deconvolution for Comprehensive Metabolome Analysis." Nature Methods 12 (6): 523-26.

Tsugawa, Hiroshi, Kazutaka Ikeda, Mikiko Takahashi, Aya Satoh, Yoshifumi Mori, Haruki Uchino, Nobuyuki Okahashi, et al. 2020. "A Lipidome Atlas in MS-DIAL 4." Nature Biotechnology 38 (10): 1159-63.

Tsugawa, Hiroshi, Ryo Nakabayashi, Tetsuya Mori, Yutaka Yamada, Mikiko Takahashi, Amit Rai, Ryosuke Sugiyama, et al. 2019. "A Cheminformatics 
Approach to Characterize Metabolomes in Stable-Isotope-Labeled Organisms." Nature Methods 16 (4): 295-98.

Versluis, Frank, Jens Voskuhl, Bartjan van Kolck, Harshal Zope, Marien Bremmer, Tjerk Albregtse, and Alexander Kros. 2013. "In Situ Modification of Plain Liposomes with Lipidated Coiled Coil Forming Peptides Induces Membrane Fusion." Journal of the American Chemical Society 135 (21): 8057-62.

Wickham, H. 2009. Ggplot2: Elegant Graphics for Data Analysis. Springer New York. Wojcieszyn, J W, R A Schlegel, K Lumley-Sapanski, and K A Jacobson. 1983. "Studies on the Mechanism of Polyethylene Glycol-Mediated Cell Fusion Using Fluorescent Membrane and Cytoplasmic Probes." Journal of Cell Biology 96 (1): 151-59.

Yang, Jian, Azadeh Bahreman, Geert Daudey, Jeroen Bussmann, René C. L. Olsthoorn, and Alexander Kros. 2016. "Drug Delivery via Cell Membrane Fusion Using Lipopeptide Modified Liposomes." ACS Central Science 2 (9): 621-30.

Yang, Jian, Yasuhito Shimada, René C. L. Olsthoorn, B. Ewa Snaar-Jagalska, Herman P. Spaink, and Alexander Kros. 2016. "Application of Coiled Coil Peptides in Liposomal Anticancer Drug Delivery Using a Zebrafish Xenograft Model." ACS Nano 10 (8): 7428-35.

Zacchetti, Boris, Joost Willemse, Brand Recter, Dino van Dissel, Gilles P. van Wezel, H. A. B. Wösten, and Dennis Claessen. 2016. "Aggregation of Germlings Is a Major Contributing Factor towards Mycelial Heterogeneity of Streptomyces." Scientific Reports 6 (1): 27045.

Zito, Francesca, Nadia Lampiasi, Igor Kireev, and Roberta Russo. 2016. "United We Stand: Adhesion and Molecular Mechanisms Driving Cell Fusion across Species." European Journal of Cell Biology 95 (12): 552-62.

Zope, Harshal R., Frank Versluis, Anita Ordas, Jens Voskuhl, Herman P. Spaink, and Alexander Kros. 2013. "In Vitro and In Vivo Supramolecular Modification of Biomembranes Using a Lipidated Coiled-Coil Motif." Angewandte Chemie International Edition 52 (52): 14247-51. 